\title{
Simulation of pelvic osteotomies applied for DDH treatment in pediatric patients using piglet models
}

\author{
Vasyl Suvorov ${ }^{1, A-D}$, Viktor Filipchuk ${ }^{1, C, E, F}$, Vadym Mazevich, ${ }^{2, B, C, E}$, Leonid Suvorov ${ }^{3, A-C}$ \\ ${ }^{1}$ Department of Joint Diseases in Children and Adolescents, The Institute of Traumatology and Orthopedics of the NAMS Ukraine, Kyiv, Ukraine \\ ${ }^{2}$ Department of Functional Diagnostics with Radiology Group, The Institute of Traumatology and Orthopedics of the NAMS Ukraine, Kyiv, Ukraine \\ ${ }^{3}$ Surgical Department, Bolgrad Central District Hospital, Ukraine \\ A - research concept and design; B - collection and/or assembly of data; C - data analysis and interpretation; \\ $D$ - writing the article; $E$ - critical revision of the article; $F$ - final approval of the article
}

\section{Address for correspondence}

Vasyl Suvorov

E-mail:vasil_suvorov@ukr.net

Funding sources

None declared

Conflict of interest

None declared

Received on May 5, 2021

Reviewed on June 20, 2021

Accepted on July 27, 2021

Published online on September 22, 2021

\section{Abstract}

Background. Developmental dysplasia of the hip (DDH) is a common hip joint pathology seen in the pediatric orthopedist's practice. Pelvic osteotomies are the reliable surgical option for DDH treatment in walking patients, and 3 osteotomies (Salter, Dega and Pemberton) are widely used in patients under 6 years of age. Plastic changes in hinge points occur during iliac fragment movement, after the performed osteotomy. The locations of these points are described in the literature, but some debate still exists about their true positions.

Objectives. To reveal hinge point locations during a simulation of pelvic osteotomies on biological models.

Materials and methods. Eighteen piglet pelvis complexes were obtained and separated according to their age. Pelvic osteotomies were simulated, and bone changes were assessed on computed tomography (CT) scans after the performed surgeries.

Results. No bone changes were found after Salter osteotomy in younger piglets, while contralateral pubic bone metaphyseal fractures were found in older animals. After Pemberton osteotomy, greenstick fractures in iliac and pubic bones metaphyses in the triradiate cartilage area were revealed in younger and older piglets. After Dega osteotomy, a posterior medial cortical layer fracture of the uncut iliac bone in the greater sciatic notch was found in all piglets. In older piglets, an additional hinge point was detected in the ipsilateral pubic bone metaphysis.

Conclusions. It was found that the age of the piglets has an impact on hinge point number and location, and this may be explained by an age-related decrease in pelvic bone and cartilage plasticity. The results of this study may help surgeons to decrease the number of preventable complications during pelvic osteotomies.

Key words: developmental dysplasia of the hip, pelvic osteotomy, hinge point, piglet model

Cite as

Suvorov V, Filipchuk V, Mazevich V, Suvorov L. Simulation of pelvic osteotomies applied for DDH treatment in pediatric patients using piglet models. Adv Clin Exp Med. 2021;30(10):1085-1090. doi:10.17219/acem/140548

DOI

10.17219/acem/140548

Copyright

Copyright by Author(s)

This is an article distributed under the terms of the

Creative Commons Attribution 3.0 Unported (CC BY 3.0)

(https://creativecommons.org/licenses/by/3.0/) 


\section{Background}

Developmental dysplasia of the hip (DDH) is one of the most common pathologies seen in the pediatric orthopedist's practice and the second most common after transient synovitis. ${ }^{1,2}$ Untreated DDH can cause hip subluxation and dislocation, hip instability, limp gait, walking delay, leg shortening, pain, and the restriction of hip movement. It also leads to early-onset hip arthritis, and about $50 \%$ of patients under 50 need a hip replacement due to the presence of residual acetabular dysplasia. ${ }^{3}$ Therefore, the timely and effective treatment of DDH is important.

In younger patients, DDH is successfully managed with nonsurgical methods, including abduction braces, closed reduction and casting. ${ }^{4}$ In patients who have started walking, surgical treatment is more effective. ${ }^{5}$ When comparing different types of surgeries (isolated open reduction, proximal femoral osteotomies, pelvic osteotomies, or single-stage surgery), the best results may be achieved after pelvic osteotomies or single-stage surgeries. ${ }^{6}$ Thus, the importance of pelvic osteotomies in terms of DDH surgical treatment is evident. Three pelvic osteotomies (Salter, Dega and Pemberton) are widely used for DDH treatment in pediatric patients under 6 years of age, and these surgeries allow for further development of the acetabulum. These procedures can be divided into complete (Salter) and incomplete (Dega and Pemberton) osteotomies of the iliac bone. ${ }^{7}$

Acetabular deformity correction during pelvic osteotomies occurs due to the acetabular reorientation after iliac bone fragment movement. Plastic changes in the hinge points take place during iliac fragment movement. The locations of hinge points are described in literature and include the symphysis pubis cartilage during Salter osteotomy, a posterior-medial cortical layer of the uncut iliac bone in the greater sciatic notch region during Dega osteotomy, and the triradiate cartilage during Pemberton osteotomy. ${ }^{8-11}$ The location of the hinge points reflects biomechanical changes that occur during pelvic osteotomy. Thus, it is important for a surgeon to know the true hinge point locations in terms of the surgical technique, preoperative planning and prevention of complications. However, there are no biomechanical or clinical studies to confirm the true hinge point locations during the abovementioned pelvic osteotomies.

Moreover, there is some debate about hinge point locations. Regarding the Salter pelvic osteotomy, the symphysis pubis cartilage has been suggested as a hinge poin. However, later studies have revealed that greenstick fractures take place in the long bone metaphysis during the loading of a shaft epiphysis connection. ${ }^{12}$ If pelvic bones are treated as long bones with areas of primary and secondary ossification, ${ }^{13}$ the pubis bone metaphyses are more likely the hinge points. The Dega osteotomy is also a subject for discussion in terms of the number and location of hinge points. While it has been suggested that the posterior medial cortical layer of the uncut iliac bone in the greater sciatic notch is a hinge point, others have described the presence of additional hinge points. ${ }^{8}$ Regarding the Pemberton osteotomy, the hinge point is considered to be located inside the triradiate cartilage, but the triradiate cartilage has 3 limbs and there is no clarification as to which limb is overloaded. ${ }^{9-11}$

As an independent factor, the patient's age may have an impact on hinge point numbers and locations. Hypothetically, younger patients may have a lower density of pelvic bones and cartilages, and greater plasticity of these tissues. This may lead to different biomechanical changes during pelvis osteotomies, and to different outcomes in younger and older patients (as described earlier). ${ }^{14}$

The true hinge point locations during pelvic osteotomies may be evaluated with different types of pelvic models. ${ }^{15-17}$ Among animals, the ideal non-rodent candidate for medical experiments is the pig. ${ }^{18}$ In this study, piglet models were used. The advantages of the piglet models for pelvic osteotomy simulation are the similarity to human anatomy and physiology, and the relatively large size of the pelvic organs. ${ }^{19}$

\section{Objectives}

The aim of this study was to assess the true hinge point locations during 3 pelvic osteotomies applied in pediatric patients for $\mathrm{DDH}$ treatment, and to reveal the impact of the patient's age on hinge point number and location.

\section{Materials and methods}

The evaluation of the hinge point locations during different pelvic osteotomies was performed using piglet pelvic complex models. The permission of the institutional ethics committee was obtained for pelvic complex model analysis and surgical simulation using these models (approval No. 2 of April 5, 2021). All piglets died from natural causes, no interventions were performed on live animals, and they were not slaughtered for the sake of this experiment.

The pelvic complexes consisted of pelvic bones, lumbar vertebrae, hip joints, proximal parts of the femoral bones, and surrounding soft tissues. All pelvic complexes were free of trauma or other injuries to the pelvis girdles or hip joints. All piglets included in this study were Landrace breeds. After death, the pelvic complexes were extracted for further examination.

The correlation between children's and piglets' chronological ages was calculated according to data available in the literature. It is known that sexual maturity in pigs occurs at 5-6 months, ${ }^{20}$ the closure of growth plates occurs at 20 months, and the age of 3-4 months in pigs is equivalent to 6-7 years of age in humans. ${ }^{21}$ For these reasons, the piglets chosen for this study were younger than 3 months (which is equivalent to a six-year-old human 
- the period of the most active acetabular development when the abovementioned pelvic osteotomies are widely used). ${ }^{22}$ We selected 18 pelvic complexes for further investigation, with 6 complexes intended for each pelvic osteotomy procedure (Salter, Dega and Pemberton). Among the 6 pelvic complexes utilized in each procedure, 3 complexes were obtained from piglets younger than 1 month and 3 from 2-2.5-month-old piglets. This was done to simulate surgeries in younger and older patients.

The pelvic complexes were frozen at $-20^{\circ} \mathrm{C}$ from the moment of obtaining until the day before the surgery. On that day, the pelvic complexes were slowly thawed at room temperature. It is known that 1 freeze-thaw cycle does not affect the biological and plastic properties of bones and cartilages, ${ }^{23-26}$ so the surgery simulation on thawed pelvic complexes was similar to those performed on live piglets. The Salter, Dega and Pemberton pelvic osteotomies were performed on these complexes, according to the original descriptions of these surgeries. ${ }^{8,26,27}$

Bone changes after the performed osteotomies were assessed using computed tomography (CT) scans (performed with a Philips Brilliance 16 CT scanner (Philips, Amsterdam, the Netherlands)). For better visualization, the slice thickness was set at $0.5 \mathrm{~mm}$ for younger piglets (up to 1 month of age) and $0.8 \mathrm{~mm}$ for older animals (2-2.5-month-old). The assessment of bone changes was performed taking into account the concept of metaphyseal lesions during shaft epiphysis load application, according to the theory presented by Thompson et al. ${ }^{12}$ It is known that all pelvic bones during their development have primary and secondary ossification centers and cartilages between them, which are equated to the metaphyses and epiphyses in long bones. ${ }^{13}$

Medixant RadiAnt DICOM Viewer software, v. 2020.1 (https://www.radiantviewer.com, accessed March 9, 2020) was used to evaluate the CT scans. The localization of bone changes was determined based on the consensus of the authors.

\section{Results}

After the performed pelvic osteotomies and the analysis of bone changes, several changes were detected on $\mathrm{CT}$ scans, according to the piglet's age and performed surgery (Table 1).

No bone changes were found after Salter pelvic osteotomy in three-week-old piglets. However, there was a metaphyseal contralateral pubic bone fracture in the symphysis pubis region in piglets aged 4 weeks and older (Fig. 1). Extrapolating these data to humans, we can assume that the hinge point in younger patients will be located

Table 1. Bone changes after performed pelvic osteotomies according to the piglet's age and performed surgery

\begin{tabular}{|c|c|c|c|}
\hline No. & Piglet's age & $\begin{array}{l}\text { Pelvic } \\
\text { osteotomy }\end{array}$ & Bone changes \\
\hline 1 & 3 weeks & Salter & none \\
\hline 2 & 3 weeks & Salter & none \\
\hline 3 & 4 weeks & Salter & contralateral pubic bone metaphysis in the symphysis pubis region \\
\hline 4 & 2 months & Salter & contralateral pubic bone metaphysis in the symphysis pubis region \\
\hline 5 & 2 months & Salter & contralateral pubic bone metaphysis in the symphysis pubis region \\
\hline 6 & 2.5 months & Salter & contralateral pubic bone metaphysis in the symphysis pubis region \\
\hline 7 & 3 weeks & Pemberton & $\begin{array}{l}\text { 1) uncut iliac bone metaphysis in the triradiate cartilage region (between iliac and pubic bones) } \\
\text { 2) medial cortical layer of the uncut iliac bone }\end{array}$ \\
\hline 8 & 3 weeks & Pemberton & $\begin{array}{l}\text { 1) uncut iliac bone metaphysis in the triradiate cartilage region (between iliac and pubic bones) } \\
\text { 2) pubic bone metaphysis in triradiate cartilage region }\end{array}$ \\
\hline 9 & 3 weeks & Pemberton & uncut iliac bone metaphysis in the triradiate cartilage region (between iliac and pubic bones) \\
\hline 10 & 2 months & Pemberton & $\begin{array}{l}\text { 1) uncut iliac bone metaphysis in the triradiate cartilage region (between iliac and pubic bones) } \\
\text { 2) pubic bone metaphysis in triradiate cartilage region }\end{array}$ \\
\hline 11 & 2 months & Pemberton & pubic bone metaphysis in triradiate cartilage region \\
\hline 12 & 2 months & Pemberton & $\begin{array}{l}\text { 1) uncut iliac bone metaphysis in the triradiate cartilage region (between iliac and pubic bones) } \\
\text { 2) pubic bone metaphysis in triradiate cartilage region }\end{array}$ \\
\hline 13 & 3 weeks & Dega & posterior medial cortical layer of the uncut iliac bone in the greater sciatic notch region \\
\hline 14 & 3 weeks & Dega & posterior medial cortical layer of the uncut iliac bone in the greater sciatic notch region \\
\hline 15 & 4 weeks & Dega & posterior medial cortical layer of the uncut iliac bone in the greater sciatic notch region \\
\hline 16 & 2 months & Dega & posterior medial cortical layer of the uncut iliac bone in the greater sciatic notch region \\
\hline 17 & 2 months & Dega & $\begin{array}{l}\text { 1) posterior medial cortical layer of the uncut iliac bone in the greater sciatic notch region } \\
\text { 2) ipsilateral pubic bone metaphysis in the symphysis pubis region }\end{array}$ \\
\hline 18 & 2 months & Dega & $\begin{array}{l}\text { 1) posterior medial cortical layer of the uncut iliac bone in the greater sciatic notch region } \\
\text { 2) ipsilateral pubic bone metaphysis in the symphysis pubis region }\end{array}$ \\
\hline
\end{tabular}




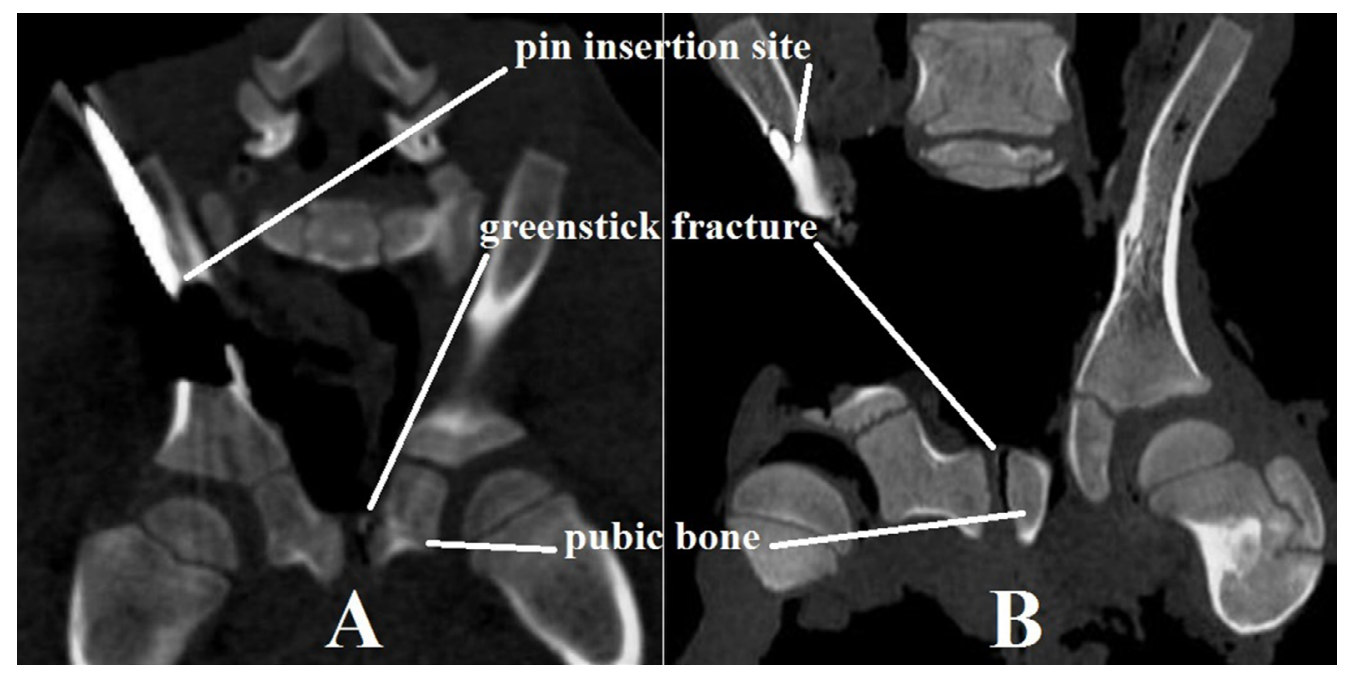

Fig. 1. Bone changes after Salter osteotomy. Contralateral pubic bone metaphyseal fracture in four-week (A) and two-month (B) old piglets

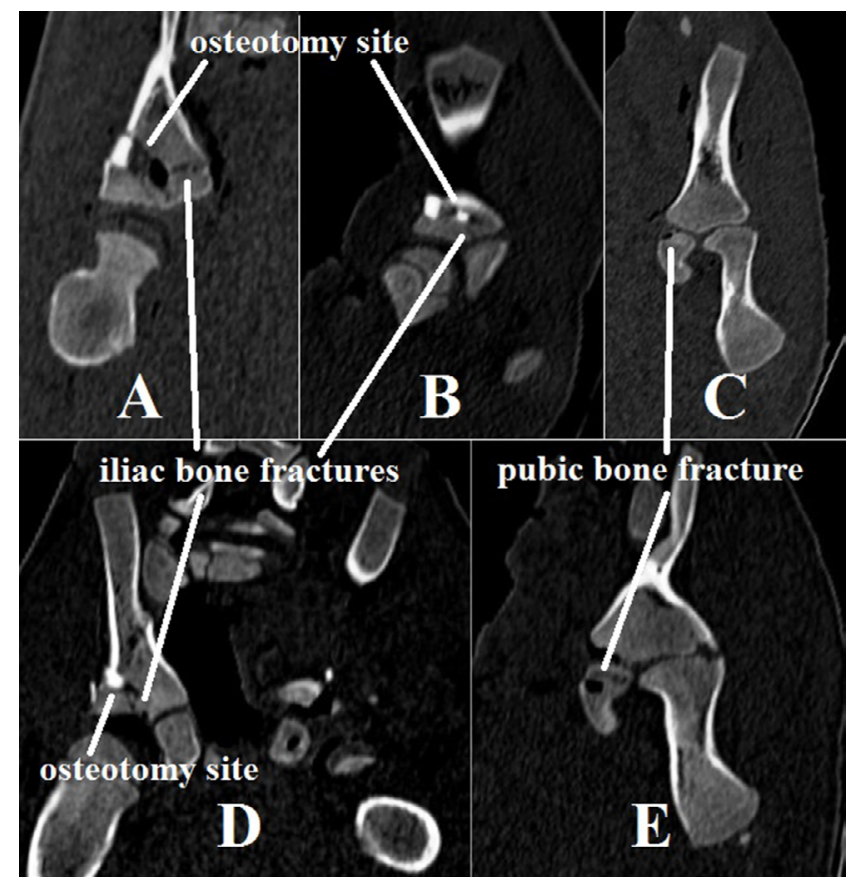

Fig. 2. Bone changes after Pemberton osteotomy. Bone changes in threeweek-old piglets. A. Fracture in the uncut iliac bone medial cortical layer B. Fracture of the iliac bone uncut part in the triradiate cartilage region; C. Pubic bone metaphyseal fracture in the triradiate cartilage region Bone changes in two-month-old piglets; D. Fracture of the iliac bone uncut part in the triradiate cartilage region; E. Pubic bone metaphyseal fracture in the triradiate cartilage region

in the symphysis pubis cartilage, which is considered to be the "classic" hinge point described earlier. However, in older patients, this hinge point is likely to be located in the contralateral pubic bone metaphysis. This may be explained by the decrease in cartilage tissue amount in the pelvis and by the increase of pelvic bone and cartilage density with the patient's age (which leads to transferring the load from the cartilaginous part of the symphysis pubis to the contralateral pubic bone metaphysis).

After Pemberton osteotomy, a greenstick metaphyseal fracture of an uncut iliac bone in the triradiate cartilage region (between iliac and pubic bones) was detected in all three-week-old piglets. In 1 piglet, a greenstick fracture was found in the medial cortical layer of the uncut iliac bone. In another piglet, a pubic bone metaphyseal fracture was found in the triradiate cartilage region (between the iliac and pubic bones). In two-month-old piglet models, a greenstick metaphyseal fracture of an uncut iliac bone was detected in the triradiate cartilage region (between iliac and pubic bones) and, in 2 piglet models, a pubic bone metaphyseal fracture in the triradiate cartilage area (between the iliac and pubic bones) was found (Fig. 2).

A fracture in the posterior medial cortical layer of the uncut iliac bone in the greater sciatic notch region was found in all piglets after the Dega osteotomy. In 2 two-month-old piglets, there was an ipsilateral pubic bone metaphyseal fracture (Fig. 3). The presence of an additional hinge point in elderly patients may be explained by an age-related increase in pelvic bone and cartilage density, which requires an additional hinge point for acetabular deformity correction during iliac fragment movement.

\section{Discussion}

$\mathrm{DDH}$ is the second most common hip disorder in children, after transient synovitis. Nonsurgical treatment of DDH is effective in non-walking patients, but in older individuals (those who have started walking), surgical treatment is preferable. Among all of the methods for surgical treatment, pelvic osteotomies are considered to be the best choice. There are 3 widely used pelvic osteotomies for DDH treatment in patients under 6 years of age - Salter, Dega and Pemberton (which involve complete or incomplete cutting of the iliac bone). Acetabular deformity correction occurs due to acetabular reorientation after iliac fragment movement, and plastic changes in hinge points are observed during these changes. The hypothetical locations of these points have been described in the literature, 


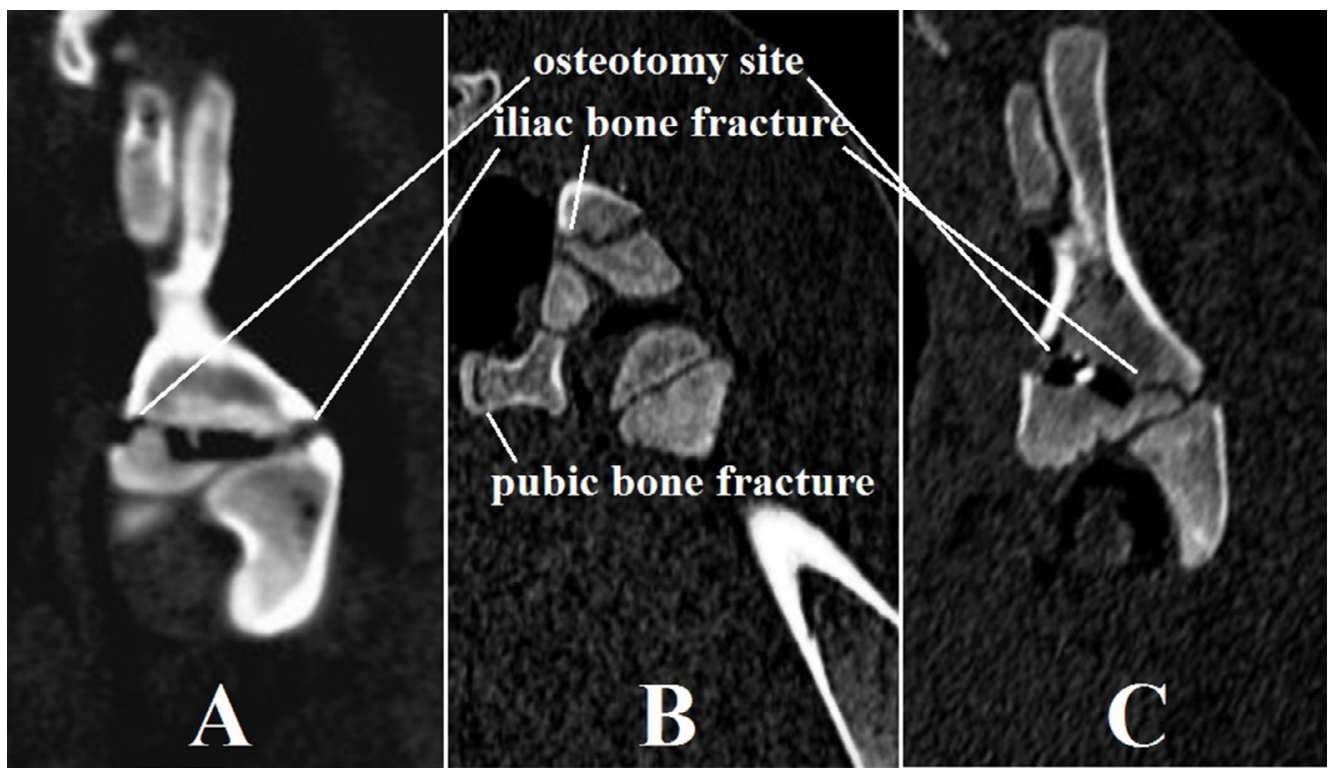

Fig. 3. Bone changes after Dega osteotomy. Fracture in the posterior medial cortical layer of the uncut iliac bone in the greater sciatic notch in three-week (A) and two-month old $(B, C)$ piglets; B. Ipsilateral pubic bone metaphyseal fracture in a twomonth-old piglet

but are still debated. ${ }^{8-9,12}$ It is essential to know the true hinge point locations for surgeons to be able to improve preoperative planning and avoid complications.

In this study, it was found that Salter pelvic osteotomy did not lead to any bone changes in younger piglets, but, in older animals, the contralateral pubic bone metaphyseal fractures were found. This confirms the Thompson's et al. hypothesis regarding metaphyseal changes during load application at the epiphysis-diaphysis border. ${ }^{12}$ However, in older individuals, the hinge point will likely be located in the contralateral pubic bone metaphysis, in the symphysis pubis region. This finding has not been described previously and it puts the conventional location of the hinge point into question. Also, due to the location of the hinge point in the contralateral hemipelvis, simultaneous bilateral application of this technique, or in combination with other pelvic osteotomies, is impossible in older patients due to the presence of pelvic ring violation, contradictory to previous data. ${ }^{28-30}$

After the Pemberton osteotomy, a greenstick metaphyseal fracture of an uncut iliac bone in the triradiate cartilage region was found in all pelvic models. The fact that the Pemberton osteotomy leads to a pubic bone metaphyseal fracture in the triradiate cartilage region indicates an overload of the triradiate cartilage limb between the iliac and pubic bones, which was not described previously. Also, this observation indicates possible triradiate cartilage damage during this surgical technique, and this damage may lead to further acetabular development disturbance, as described by others. ${ }^{31,32}$ Therefore, the Pemberton pelvic osteotomy should be used with caution, especially in patients under 6 years of age (during the period of active acetabular development). ${ }^{22}$

The hinge point location after Dega osteotomy was found in the posterior medial cortical layer of the uncut iliac bone in the greater sciatic notch region. This confirms the conventional concept described by the author of this technique. Thus, the acetabular deformity should be carefully corrected during this technique to avoid unnecessary fracture of the intact part of the iliac bone. In older piglets, an additional hinge point was found in the ipsilateral pubic bone metaphysis in the symphysis pubis region, which confirms the data of Czubak et al. regarding the presence of 2 hinge points in this technique. ${ }^{8}$ This observation indicates that simultaneous bilateral Dega osteotomy, or the combination of this procedure with other pelvic osteotomies is impossible in older patients, due to the presence of pelvic ring violation.

\section{Limitations}

For surgery simulation and bone changes assessment, piglet pelvic complexes were used instead of human ones. Thus, the postsurgical results may not be the same as in humans. In addition, the bone changes in this study were assessed using healthy pelvis models rather than ones with DDH. No cartilage changes were assessed after the simulated surgeries. Therefore, the conclusions about triradiate cartilage overload were drawn indirectly. Also, a small number of pelvises was included in this investigation and future studies should be planned with a larger number of cases.

\section{Conclusions}

The surgeon must know the true hinge point locations and their number during different pelvic osteotomies applied for DDH treatment in pediatric patients. The current data indicate that the patient's age may have an impact on hinge point locations and number. The results of the current work may help pediatric surgeons to improve surgical planning and avoid some preventable complications. 


\section{ORCID iDs}

Vasyl Suvorov (10 https://orcid.org/0000-0002-0862-7997 Viktor Filipchuk (1) https://orcid.org/0000-0002-9727-9532 Vadym Mazevich (1) https://orcid.org/0000-0002-3617-5043 Leonid Suvorov (1) https://orcid.org/0000-0002-8744-1751

\section{References}

1. Storer SK, Skaggs DL. Developmental dysplasia of the hip. Am Fam Physician. 2006;74(8):1310-1316. PMID:17087424

2. Neville DNW, Zuckerbraun N. Pediatric nontraumatic hip pathology. Clin PediatrEmerg Med. 2016;17(1):13-28. doi:10.1016/j.cpem.2016.01.005

3. Clohisy JC, Dobson MA, Robison JF, et al. Radiographic structural abnormalities associated with premature, natural hip-joint failure. J Bone Joint Surg Am. 2011;93(Suppl 2):3-9. doi:10.2106/JBJS.J.01734

4. Cooper AP, Doddabasappa SN, Mulpuri K. Evidence-based management of developmental dysplasia of the hip. Orthop Clin North Am. 2014;45(3):341-354. doi:10.1016/j.ocl.2014.03.005

5. Chen Q, Deng Y, Fang B. Outcome of one-stage surgical treatment of developmental dysplasia of the hip in children from 1.5 to 6 years old: A retrospective study. Acta Orthop Belg. 2015;81(3):375-383. PMID: 26435230

6. Kothari A, Grammatopoulos G, Hopewell S, Theologis T. How does bony surgery affect results of anterior open reduction in walkingage children with developmental hip dysplasia? Clin Orthop Relat Res. 2016;474(5):1199-1208. doi:10.1007/s11999-015-4598-x

7. Sales de Gauzy J. Pelvic reorientation osteotomies and acetabuloplasties in children: Surgical technique. Orthop Traumatol Surg Res. 2010;96(7):793-799. doi:10.1016/j.otsr.2010.07.004

8. Czubak J, Kowalik K, Kawalec A, Kwiatkowska M. Dega pelvic osteotomy: Indications, results and complications. J Child Orthop. 2018;12(4): 342-348. doi:10.1302/1863-2548.12.180091

9. Ezirmik N, Yildiz K. A biomechanical comparison between Salter innominate osteotomy and Pemberton pericapsular osteotomy. Eurasian J Med. 2012;44(1):40-42. doi:10.5152/eajm.2012.08

10. Ertürk C, Altay MA, Işikan UE. A radiological comparison of Salter and Pemberton osteotomies to improve acetabular deformations in developmental dysplasia of the hip. J Pediatr Orthop B. 2013;22(6): 527-532. doi:10.1097/BPB.0b013e32836337cd

11. Chunho C, Wang TM, Kuo KN. Pelvic osteotomies for developmental dysplasia of the hip. In: Spasovski D, ed. Developmental Diseases of the hip. IntechOpen; 2017. https://www.intechopen.com/chapters/ 54481. Accessed August 5, 2021.

12. Thompson A, Bertocci G, Kaczor K, Smalley C, Pierce MC. Biomechanical investigation of the classic metaphyseal lesion using an immature porcine model. AJR Am J Roentgenol. 2015;204(5):W503-W509. doi:10.2214/AJR.14.13267

13. Verbruggen SW, Nowlan NC. Ontogeny of the human pelvis. Anat Rec (Hoboken). 2017;300(4):643-652. doi:10.1002/ar.23541

14. El-Sayed M, Ahmed T, Fathy S, Zyton H. The effect of Dega acetabuloplasty and Salter innominate osteotomy on acetabular remodeling monitored by the acetabular index in walking $\mathrm{DDH}$ patients between 2 and 6 years of age: Short- to middle-term follow-up. J Child Orthop. 2012;6(6):471-477. doi:10.1007/s11832-012-0451-x

15. Ike $\mathrm{H}$, Inaba $\mathrm{Y}$, Kobayashi N, et al. Effects of rotational acetabular osteotomy on the mechanical stress within the hip joint in patients with developmental dysplasia of the hip: A subject-specific finite element analysis. Bone Joint J. 2015;97-B(4):492-497. doi:10.1302/0301620X.97B4.33736
16. Yassir W, Mahar A, Aminian A, Newton P, Wenger D. A comparison of the fixation stability of multiple screw constructs for two types of pelvic osteotomies. J Pediatr Orthop. 2005;25(1):14-17. doi:10.1097/ 00004694-200501000-00005

17. Fukushima K, Takahira N, Uchiyama K, Moriya M, Takaso M. Pre-operative simulation of periacetabular osteotomy via a three-dimensional model constructed from salt. SICOT J. 2017;3:14. doi:10.1051/ sicotj/2016051

18. Bassols A, Costa C, Eckersall PD, Osada J, Sabrià J, Tibau J. The pig as an animal model for human pathologies: A proteomics perspective. Proteomics Clin Appl. 2014;8(9-10):715-731. doi:10.1002/prca.201300099

19. Marchant-Forde JN, Herskin MS. Pigs as laboratory animals. In: Špinka, Camerlink I, eds. Advances in Pig Welfare. Cambridge, UK: Woodhead Publishing; 2018:445-475. doi:10.1016/b978-0-08-101012-9.00015-0

20. Reiland S. Growth and skeletal development of the pig. Acta Radiol Suppl. 1978;358:15-22. PMID:233594

21. Tohyama S, Kobayashi E. Age-appropriateness of porcine models used for cell transplantation. Cell Transplant. 2019;28(2):224-228. doi:10.1177/0963689718817477

22. Novais EN, Pan Z, Autruong PT, Meyers ML, Chang FM. Normal percentile reference curves and correlation of acetabular index and acetabular depth ratio in children. J Pediatr Orthop. 2018;38(3):163-169. doi:10.1097/BPO.0000000000000791

23. Shaw JM, Hunter SA, Gayton JC, Boivin GP, Prayson MJ. Repeated freeze-thaw cycles do not alter the biomechanical properties of fibular allograft bone. Clin Orthop Relat Res. 2012;470(3):937-943. doi:10. 1007/s11999-011-2033-5

24. Changoor A, Fereydoonzad L, Yaroshinsky A, Buschmann MD. Effects of refrigeration and freezing on the electromechanical and biomechanical properties of articular cartilage. J Biomech Eng. 2010;132(6):064502. Erratum in: J Biomech Eng. 2011;133(4):047001. doi:10.1115/1.4000991

25. Szarko M, Muldrew K, Bertram JE. Freeze-thaw treatment effects on the dynamic mechanical properties of articular cartilage. BMC Musculoskelet Disord. 2010;11:231. doi:10.1186/1471-2474-11-231

26. Shannon CE, Kelley SP. The Salter innominate osteotomy. In: Hamdy RC, Saran N, eds. Pediatric Pelvic and Proximal Femoral Osteotomies. New York, USA: Springer International Publishing; 2018:29-35. doi:10. 1007/978-3-319-78033-7_3

27. Kuo KN, Wang TM. Pemberton osteotomy (anterolateral acetabuloplasty). In: Hamdy RC, Saran N, eds. Pediatric Pelvic and Proximal Femoral Osteotomies. New York, USA: Springer International Publishing; 2018:111-119. doi:10.1007/978-3-319-78033-7_12

28. Agus H, Bozoglan M, Kalenderer Ö, Kazımoğlu C, Onvural B, Akan İ. How are outcomes affected by performing a one-stage combined procedure simultaneously in bilateral developmental hip dysplasia? Int Orthop. 2014;38(6):1219-1224. doi:10.1007/s00264-014-2330-1

29. Ezirmik N, Yildiz K. Advantages of single-stage surgical treatment with salter innominate osteotomy and Pemberton pericapsular osteotomy for developmental dysplasia of both hips. J Int Med Res. 2012; 40(2):748-755. doi:10.1177/147323001204000240

30. Li L, Yang X, Song B, Jiang J, Yang L, Tang X. Biomechanical investigation of pelvic stability in developmental dysplasia of the hip: Unilateral salter osteotomy versus one-stage bilateral salter osteotomy. J Orthop Surg Res. 2020;15(1):169. doi:10.1186/s13018-020-01683-w

31. Plaster RL, Schoenecker PL, Capelli AM. Premature closure of the triradiate cartilage: A potential complication of pericapsular acetabuloplasty. J Pediatr Orthop. 1991;11(5):676-678. PMID:1918360

32. Leet Al, Mackenzie WG, Szoke G, Harcke HT. Injury to the growth plate after Pemberton osteotomy. J Bone Joint Surg Am. 1999;81(2):169-176. doi:10.2106/00004623-199902000-00004 